\title{
Experimental Study of Longitudinal and Transverse Spin Polarization in LEED from Platinum (111)
}

\author{
Peter Bauer* and Wolfgang Eckstein \\ Max-Planck-Institut für Plasmaphysik, Euratom Association, \\ Garching bei München, Federal Republic of Germany \\ Norbert Müller \\ Universität Osnabrück, Fachbereich Physik, Osnabrück, Federal Republic of Germany \\ Received May 11, 1983
}

In LEED from the (111) surface of platinum the dependence of the spin polarization on the polar angle $\vartheta$ and the azimuthal angle $\varphi$ in the energy range from $E=60 \mathrm{eV}$ to $E$ $=70 \mathrm{eV}$ was investigated.

Our results further confirm earlier findings concerning the symmetry properties of the polarization vector $P$. An appropriate rotation diagram clearly demonstrates that by means of momentum averaging the strong influence of multiple scattering on spin polarization cannot be eliminated. By systematic analysis of $P(E, \vartheta, \varphi)$ data it is shown that there are strong polarization features which are coupled to a fixed direction of the crystal.

Spin up/spin down partial intensities evaluated from simultaneously measured spin polarization and total intensity data show quite different behaviour. Therefore, if spin dependent scattering is strong, the total intensity cannot be calculated correctly within a theory, which handles the multiple scattering spin-independent.

\section{Introduction}

Seven years ago the first experimental studies of spin polarization phenomena in LEED were reported for $\mathrm{W}(001)$ and $\mathrm{Au}(110)$ surfaces [1-3]. Up to now a great number of experiments have been performed using different experimental techniques, with $\mathrm{W}(001)$, $\mathrm{Au}(110), \mathrm{Pt}(111)$ and $\mathrm{Ni}(111)$ and $\mathrm{Fe}(110)$ as crystal surfaces for diffraction. These experiments, which are summed up in the review articles of Feder [4] and Pierce [5] (except for the Fe(110) measurements [6]) have shown that the spin polarization which arises for non-magnetic materials from spin-orbit coupling is nearly always governed by multiple electron scattering.

The aim of this contribution is to show that by analysis of the spin polarization in a sufficiently large range of the experimental parameters, i.e. the energy

* Present address: Universität Linz, Institut für Experimentalphysik, A-4040 Linz-Auhof, Austria
$E$, the polar angle $\vartheta$ and the azimuthal angle $\varphi$, one can obtain information on the nature of the multiple scattering processes which lead to the pronounced polarization effects observed in LEED.

If the experiment is done using an unpolarized electron beam for diffraction, the two spin states $(\uparrow$ and $\downarrow)$ along the quantization axis $\hat{n}$, which is the normal to the scattering plane, are equally populated. This may be written as

$$
\begin{aligned}
& I_{0}^{\uparrow}=I_{0}^{\downarrow}, \\
& \mathbf{P}_{0}=\frac{I_{0}^{\uparrow}-I_{0}^{\downarrow}}{I_{0}^{\uparrow}+I_{0}^{\downarrow}} \cdot \hat{n}, \quad \hat{n}=\frac{\mathbf{k}_{0} \times \mathbf{k}}{\left|\mathbf{k}_{0} \times \mathbf{k}\right|} .
\end{aligned}
$$

In Eq. (1) $\mathbf{P}_{0}$ denotes the polarization vector of the incident beam and $\mathbf{k}_{0}$ and $\mathbf{k}$ are the momentum vectors of the incident and the outgoing beam, respectively. 
If the scattering plane, defined by the vectors $\mathbf{k}_{0}$ and $\mathbf{k}$, is a mirror plane of the crystal, the quantization axis remains unchanged [7], even in the presence of multiple scattering. The polarization vector after diffraction is then given by.

$$
\begin{aligned}
& \mathbf{P}=\frac{I^{\uparrow}-I^{\downarrow}}{I^{\uparrow}+I^{\downarrow}} \cdot \hat{n}=P_{n} \cdot \hat{n}, \\
& |\mathbf{P}|=\left|\frac{I^{\uparrow}-I^{\downarrow}}{I^{\uparrow}+I^{\downarrow}}\right|=P_{n} .
\end{aligned}
$$

In Eq. (2) $I^{\uparrow}$ and $I^{\downarrow}$ denote the spin-dependent diffracted intensities and $P$ is the degree of polarization. In this case, all information on the polarization is obtained by measuring one polarization component, namely $P_{n}$, perpendicular to the scattering plane.

When the scattering plane is not a mirror plane of the crystal, the quantization axis may change. The two polarization components parallel to the scattering plane therefore generally do not vanish owing to multiple scattering. For Pt(111), for example, it has been shown that the longitudinal component $P_{k}$, which is parallel to the momentum of the outgoing beam, may contain information [8]:

$P_{k}=\mathbf{P} \cdot \hat{k} \quad$ with $\hat{k}=\frac{\mathbf{k}}{|\mathbf{k}|}$.

The use of the Pt(111) surface has the advantage that because of the threefold symmetry of this crystal face the influence of time reversal symmetry on the polarization may be studied by measuring rotation diagrams for individual components of the polarization vector. A further important advantage of the Pt(111) surface is that it is unreconstructed at all temperatures. (The relaxation of the first layer spacing was found to be about $1 \%$ by LEED studies and by ion scattering experiments [9-14].)

The $\operatorname{Pt}(111)$ polarization data therefore play a predominant role in testing the theory $[13,14]$, which so far does not handle reconstructed surfaces. Furthermore, the simple geometry of the crystal surface may simplify interpretation of the polarization data.

\section{Experimental Setup}

The polarization measurements in LEED from $\mathrm{Pt}(111)$ reported here were done as a double scattering experiment: an unpolarized electron beam is diffracted by the Pt(111) crystal, and the resulting polarization is analyzed by a second scattering process in a thin gold foil at an electron energy of $120 \mathrm{keV}$ (Mott scattering). The diffraction system, which is

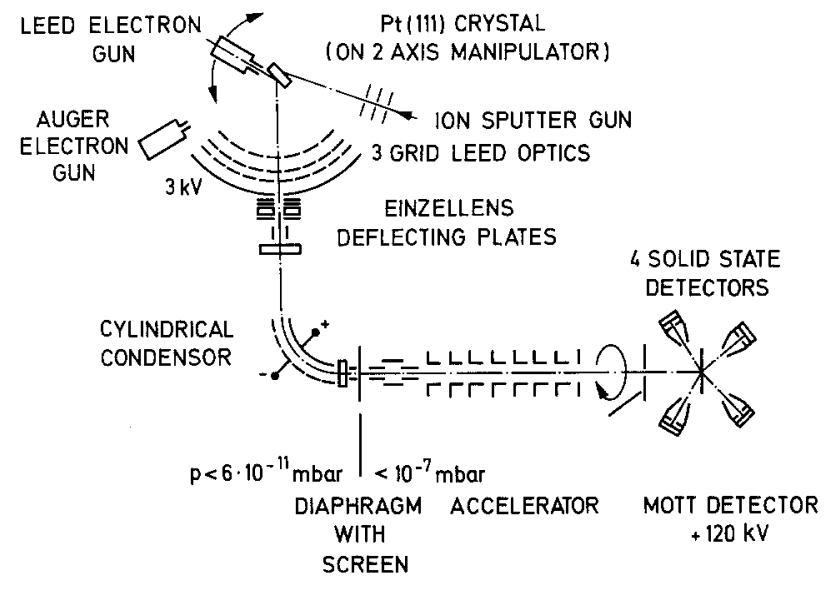

Fig. 1. Experimental setup

operated at a pressure of less than $6 \times 10^{-11}$ mbar, and the Mott scattering system, which operates at less than $10^{-7}$ mbar, are interconnected by a $90^{\circ}$ deflection unit, a diaphragm and the accelerator tube. A schematic drawing of the set up is shown in Fig. 1, a detailed description having been given earlier [15]. Only some main features will be repeated here: The Mott detector can be rotated about the axis of the incoming electron beam. This allows the polarization components $P_{n}$ and $P_{k}$ to be analyzed. The electrons, which are scattered in the Au foil, are detected by surface barrier detectors. The four-counter arrangement allows compensation of asymmetries due to misalignment of the detector setup [16]. One part of the counting electronics is put in the high-voltage region, the other is operated at ground potential. The pulses are fed to ground via electrostatically shielded pulse transformers. These pulses are counted and then recorded in a desk computer (HP9815 A) which also evaluates the polarization from these data. A schematic of the counting electronics and data processing is given in Fig. 2.

Special care has been taken to achieve a well aligned geometry in the diffraction system because the investigation of the symmetry properties of the polarization requires that the experimental data contain the symmetry expected from that of the crystal face. The optical surface of the crystal was therefore oriented to coincide with the crystallographic (111) plane within $0.1^{\circ}$, and a crystal manipulator was constructed [17], which allows precise adjustment of the crystal rotation axes with respect to the crystal surface. Furthermore, the polar rotation axis can be aligned with respect to the axis of the electron gun and, in addition, the azimuthal rotation axis of the crystal can be adjusted to be perpendicular to the polar axis. The polar angle $\vartheta$ between the surface 


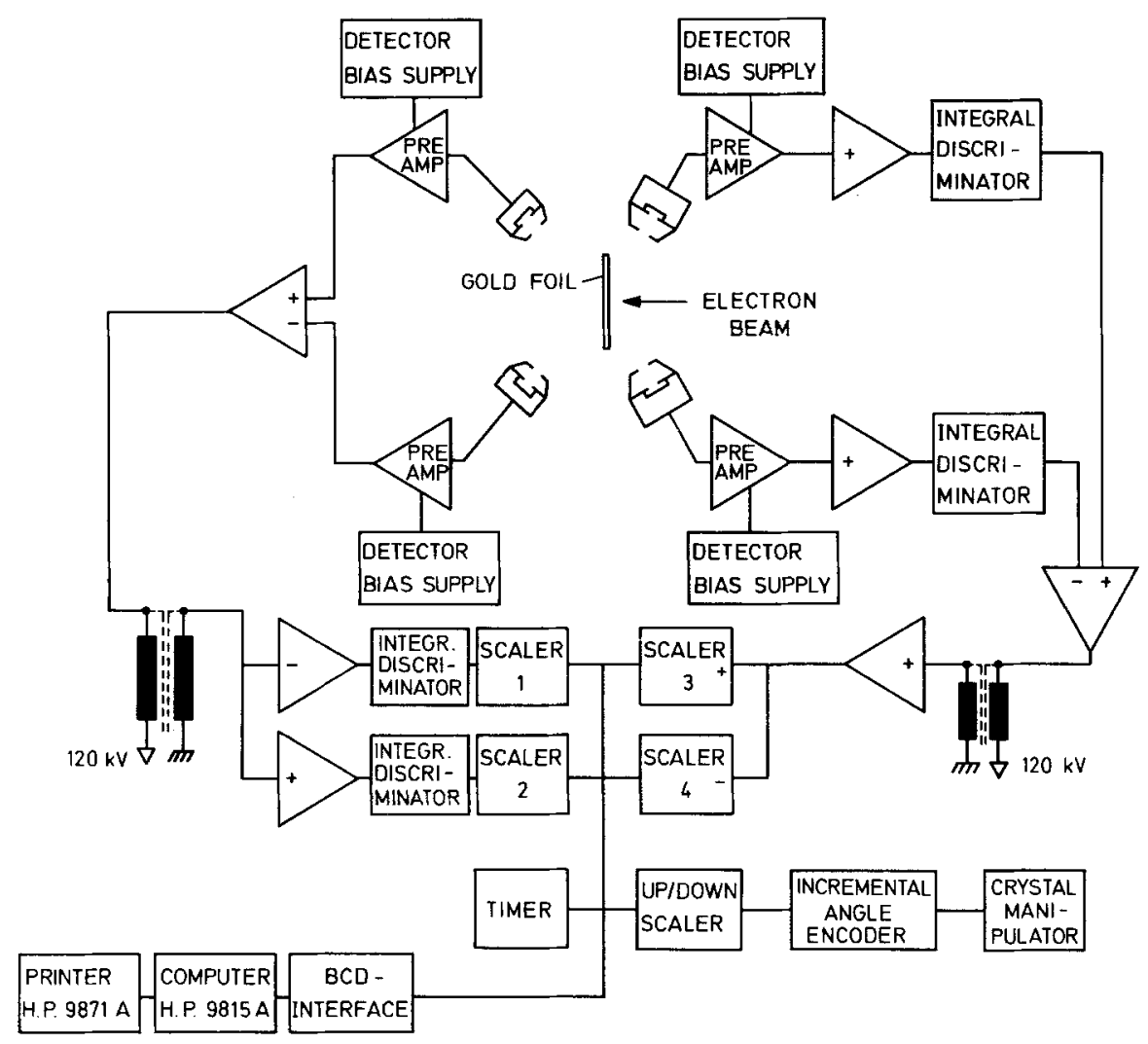

Fig. 2. Scheme of the polarization detector, the counting electronics and the data processing system

normal of the crystal and the probe-hole axis can be varied through $270^{\circ}$ and the azimuthal angle $\varphi$, whose rotation axis coincides with the surface normal (see Fig. 3), can be varied over a range of $200^{\circ}$. Both angles can be set to within $0.1^{\circ}$, which was necessary for some diffraction conditions where drastic polarization changes were observed within a few tenths of one degree for both angles $\vartheta$ and $\varphi$. The angle of acceptance of the probe-hole was therefore chosen to be about $0.1^{\circ}$.

Although the polarization also changes very rapidly as the energy varies, it was preferred to measure at fixed energy with varying angles $\vartheta$ and $\varphi$, since the energy of the incident electron beam may be reset better than $0.1 \mathrm{eV}$ more easily than the angles to $0.5^{\circ}$. The angles $\vartheta, \varphi$ may vary owing to backlash and time-dependent stray magnetic fields. Here the accuracy for absolute values of the polar angle is limited to $\pm 0.5^{\circ}$, while changes in polar angle are accurate within $\pm 0.1^{\circ}$. In addition, there are the following uncertainties: for changes of the azimuthal angle $\pm 0.4^{\circ}$, for polarization zero $\pm 1 \%$ and for the polarization sensitivity of the Mott detector $\pm 7 \%$ of the measured values. The electron energy was measured from the midpotential of the filament of the electron gun to ground potential. The energy of the electrons with respect to the crystal may differ from this energy by the contact potential between the

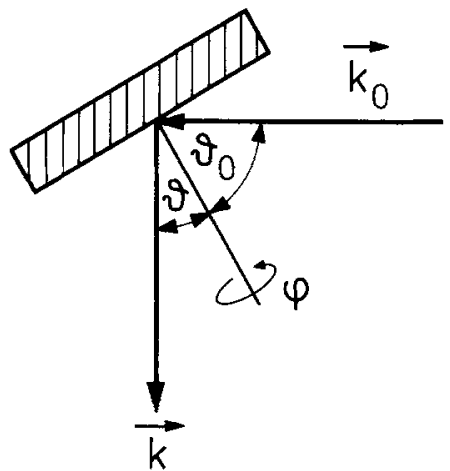

Fig. 3. The scattering geometry and the notation of the polar angles of incidence and exit, $\vartheta_{0}$ and $\vartheta$, respectively, and the azimuthal angle $\varphi \cdot \varphi=0$ was chosen for the scattering plane being parallel to the (110) plane of the crystal. $\hat{n}$ denotes the crystal surface normal

tungsten filament and the crystal, but this is less than $0.5 \mathrm{eV}$. The experimental results are given with error bars which correspond to the statistical error of the counting in the Mott detector.

\section{Experimental Results and Discussion}

The rotation diagrams published up to now have shown a strong azimuth dependence. This shows 


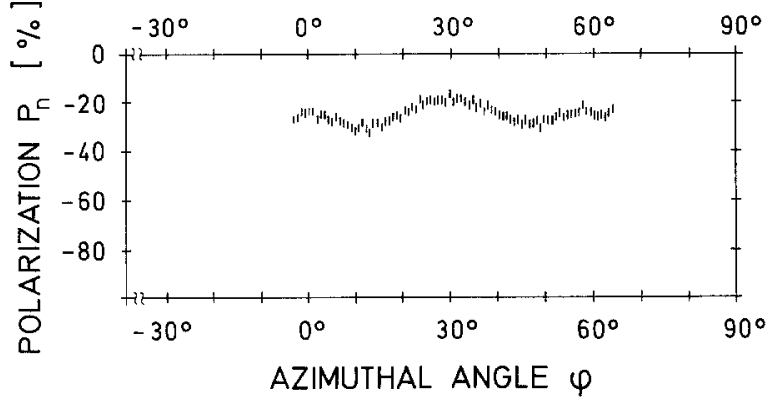

Fig. 4. Rotation diagram for the polarization component $P_{n}$ at $E$ $=70 \mathrm{eV}$ and $\vartheta=11^{\circ}$

that the kinematic description of electron diffraction $[18,19]$ is not correct. It may, however, be possible to eliminate the influence of multiple scattering by a constant momentum transfer averaging method (CMTA) [18]. Wang et al. [20] applied the CMTA procedure to measured $I(E, \vartheta)$ (intensity) and $S(E, \vartheta)$ (strength of spin-dependent scattering) data obtained at fixed azimuth and thus extracted the kinematic behaviour. On the other hand, one could try to average the polarization data of a rotation diagram. If the influence of multiple scattering could be eliminated in this way, the averaging procedure should yield the same result as electron atom scattering, corrected for the inner potential $V_{0, r}$ of the crystal:

$E^{\prime}=E+V_{0, r}$,

$\sin \vartheta^{\prime}=\sqrt{\frac{E}{E^{\prime}}} \cdot \sin \vartheta$

The notations $E^{\prime}$ and $\vartheta^{\prime}$ are used to describe the energy and the polar angle inside the crystal. The fact that this procedure will not work in general may be seen from the rotation diagram shown in Fig. 4. This $P(\varphi)$ diagram measured at an energy of $70 \mathrm{eV}$ and a polar angle of $11^{\circ}$ shows a very weak dependence of the polarization on the azimuth, the polarization varying between $-20 \%$ and $-30 \%$. Irrespective of the details of the averaging procedure, the resulting polarization value will be more negative than $-20 \%$, while the corresponding value in electron atom scattering is calculated to be $0 \%$ to $-10 \%$ [21] at an energy of $85 \mathrm{eV}$ and a polar angle of $10^{\circ}$. This calculated value is expected to be correct, and therefore the conclusion may be drawn that an averaging procedure of polarization in a single rotation diagram will not be able to eliminate the influence of multiple scattering.

As the aim of this contribution is to give a phenomenologic interpretation of our polarization results, we just try to interpret those polarization features which are sharp peaks with respect to both the
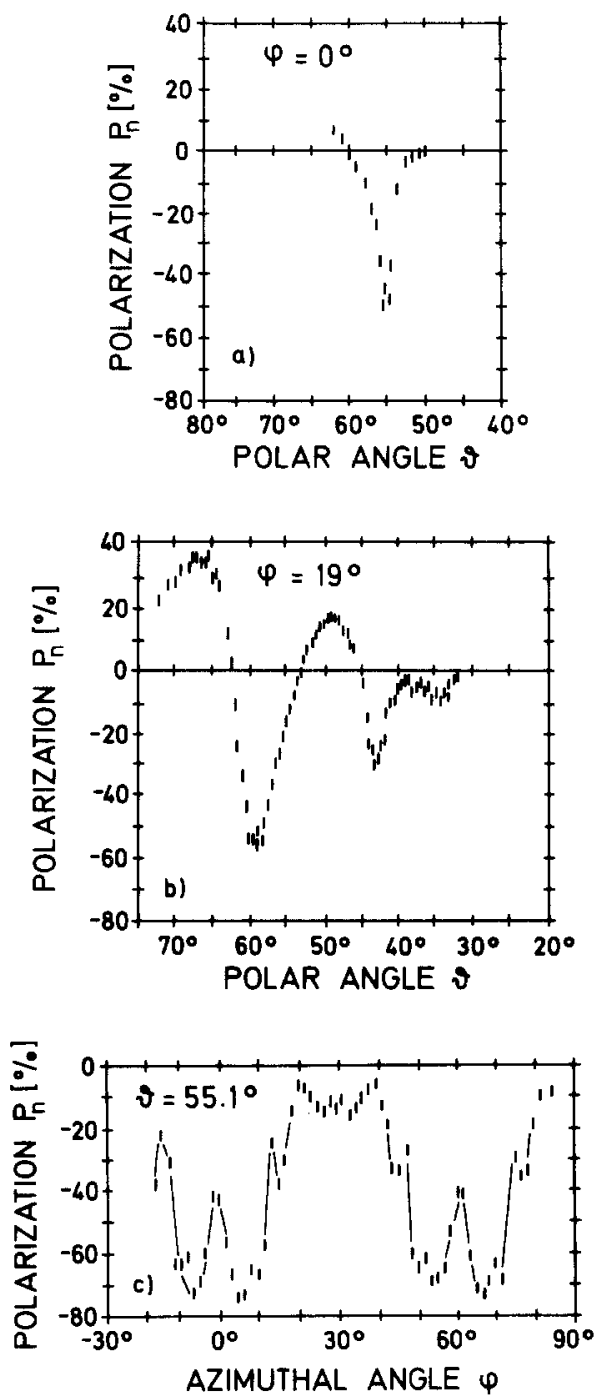

Fig. 5. The polarization component $P_{n}$ as a function of the polar angle $\vartheta$ at fixed azimuth $\varphi=0^{\circ}$ and $\varphi=19^{\circ}$ and as a function of the azimuth for $\vartheta=55,1$ for $E=65 \mathrm{eV}$

energy and the diffraction geometry $(\vartheta$ and $\varphi)$, which is a severe restriction of the experimental data. Figure 5 shows an example where this condition is not fulfilled. The $P(\vartheta)$ diagram measured at $E=65 \mathrm{eV}$ and $\varphi=0^{\circ}$ (Fig. 5a) has a sharp minimum at $\vartheta=55^{\circ}$. Owing to the rotation symmetry of the crystal the polarization is expected to have an extremum also with respect to the azimuth for $\varphi=0^{\circ}$. The rotation diagram of Fig. $5 \mathrm{c}$ shows that this extremum exists, and that it is a maximum. Therefore, $\left(\vartheta=55^{\circ}, \varphi=0^{\circ}\right)$ is a saddle point over the $\vartheta-\varphi$ plane for $E=65 \mathrm{eV}$. Another set of $P(\vartheta)$ and $P(\varphi)$ curves, measured for $E$ $=60 \mathrm{eV}$, is shown in Figs. 6 and 7. These curves contain information on the symmetry properties of the polarization [22] and the multiple scattering processes. The rotation diagrams contain the ro- 


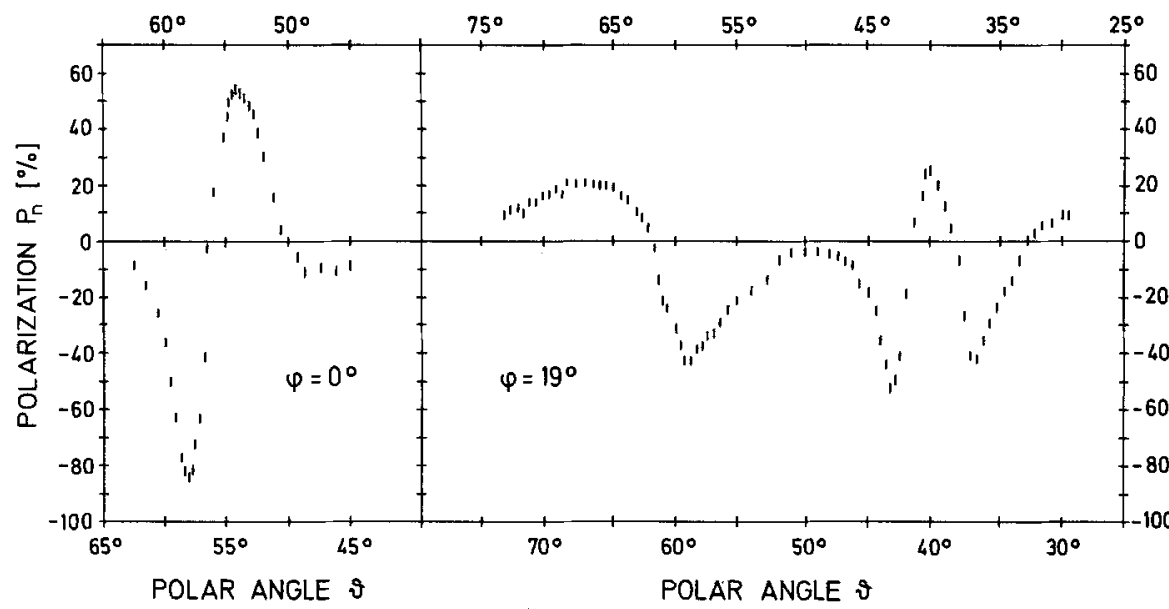

Fig. 6. The polarization component $P_{n}$ as a function of the polar angle $\vartheta$ at fixed azimuth $\varphi=0^{\circ}$ and $\varphi=19^{\circ}$
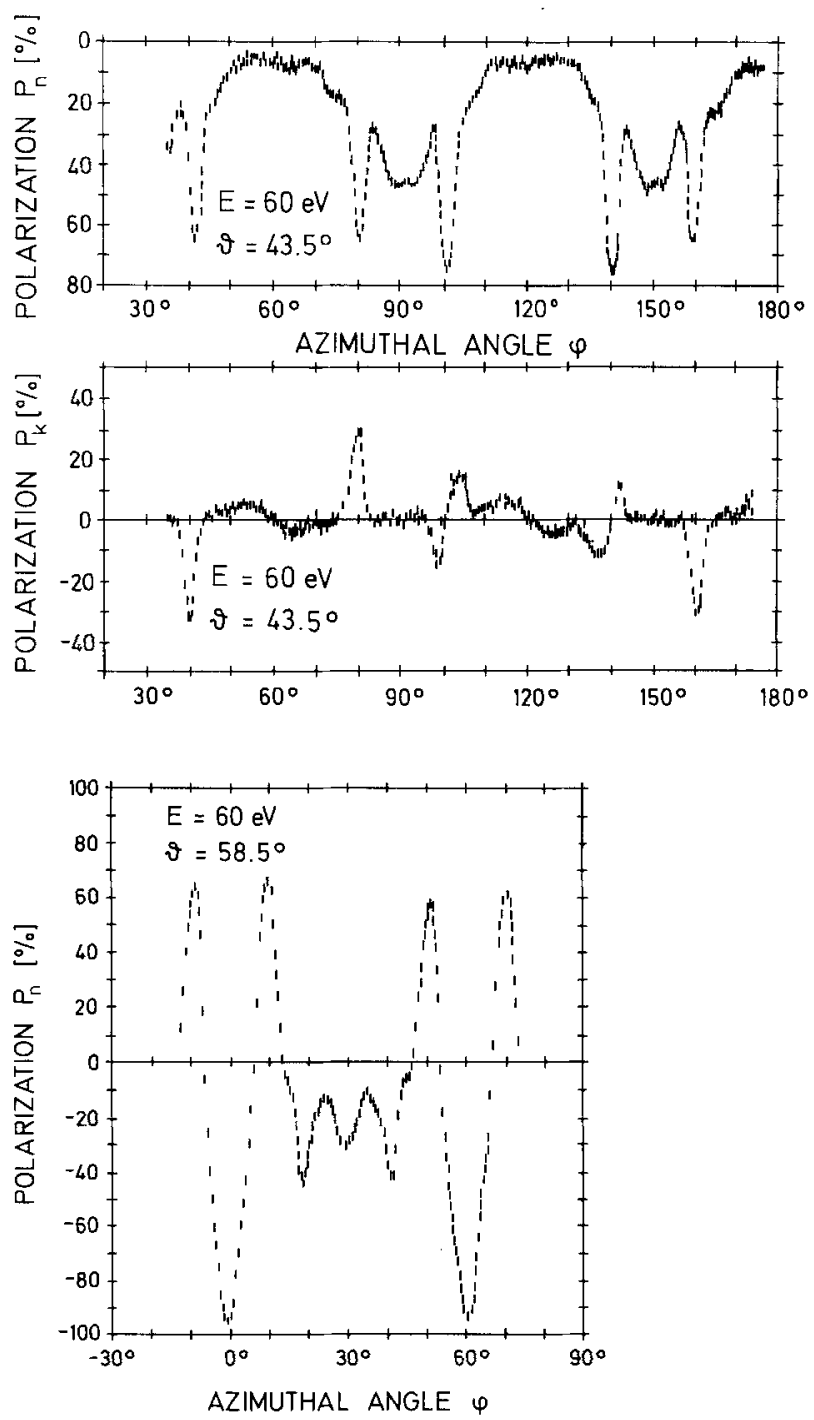

Fig. 7. Rotation diagrams of polarization components at $E$ $=60 \mathrm{eV}$ and a polar angle in the range from $\vartheta=43.5^{\circ}$ to $\vartheta=67^{\circ}$
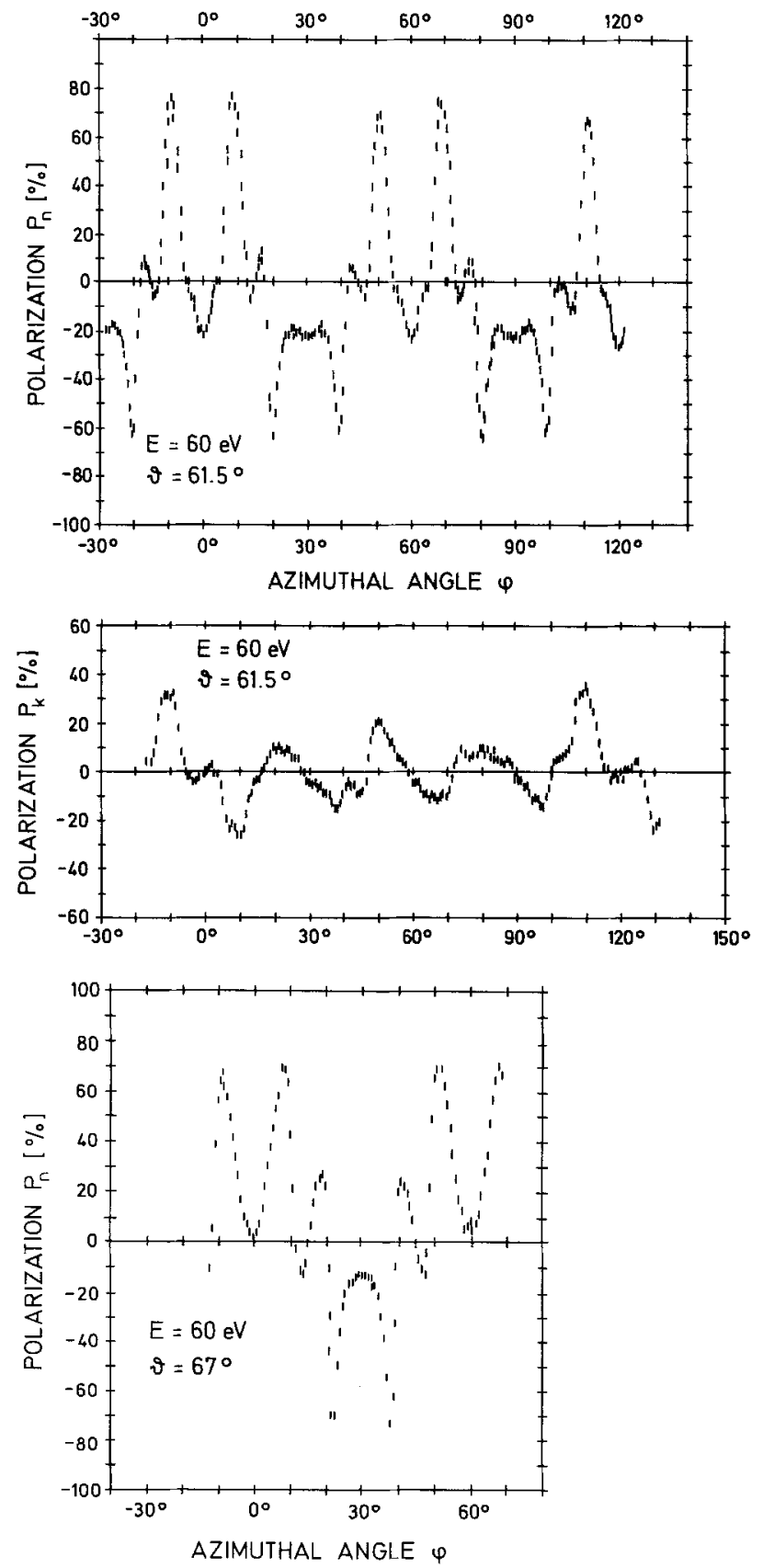


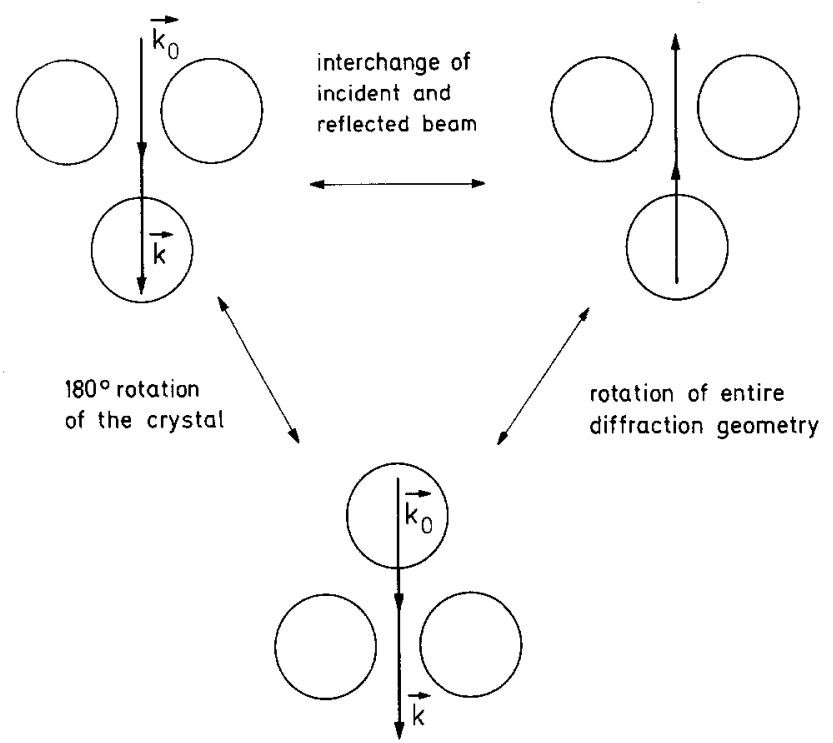

Fig. 8. Illustration of the equivalence of time reversal and an azimuthal rotation of $180^{\circ}$ for the $(00)$ beam

tation symmetry of the crystal surface which is threefold. For the measurement of the intensity and the degree of polarization in the $(00)$ beam this symmetry would be enlargened sixfold by time reversal [22-25] which is equivalent to a $60^{\circ}$ azimuthal rotation of the crystal (see for illustration Fig. 8). For the polarization components $P_{n}$ and $P_{k}$ the symmetry stays threefold, however, as has already been shown $[8,15,26]$. As regards only the $P_{n}(\varphi)$ diagrams of Fig. 7 it becomes obvious that with increasing polar angle the symmetry approaches the sixfold symmetry. This means that the multiple scattering processes within the surface plane play a more and more predominant role, perhaps owing to the reduction of the penetration depth with increasing polar angle, which means a more glancing incidence. On the other hand, the rotation diagrams of the longitudinal component $P_{k}$ show threefold symmetry up to a polar angle of $61.5^{\circ}$ owing to a selection of multiple scattering processes which include scattering out of the scattering plane. Looking at Figs. 6 and 7 it is seen that the polarization has a sharp minimum with respect to $\vartheta$ and $\varphi$ at $\vartheta=43.5^{\circ}$ and $\varphi=19^{\circ}$. This extremum is connected with a very deep minimum of the diffracted intensity. In order to investigate this polarization feature further, we measured $P(\vartheta)$ curves around $\vartheta$ $=43^{\circ}$ in the energy region between $57.5 \mathrm{eV}$ and $67.5 \mathrm{eV}$. The result is shown in Fig. 9, which shows that this polarization minimum occurs at the same polar angle in the energy range from $60 \mathrm{eV}$ to $66.5 \mathrm{eV}$, i.e. at a fixed diffraction geometry. This leads us to the conclusion that it is connected with the

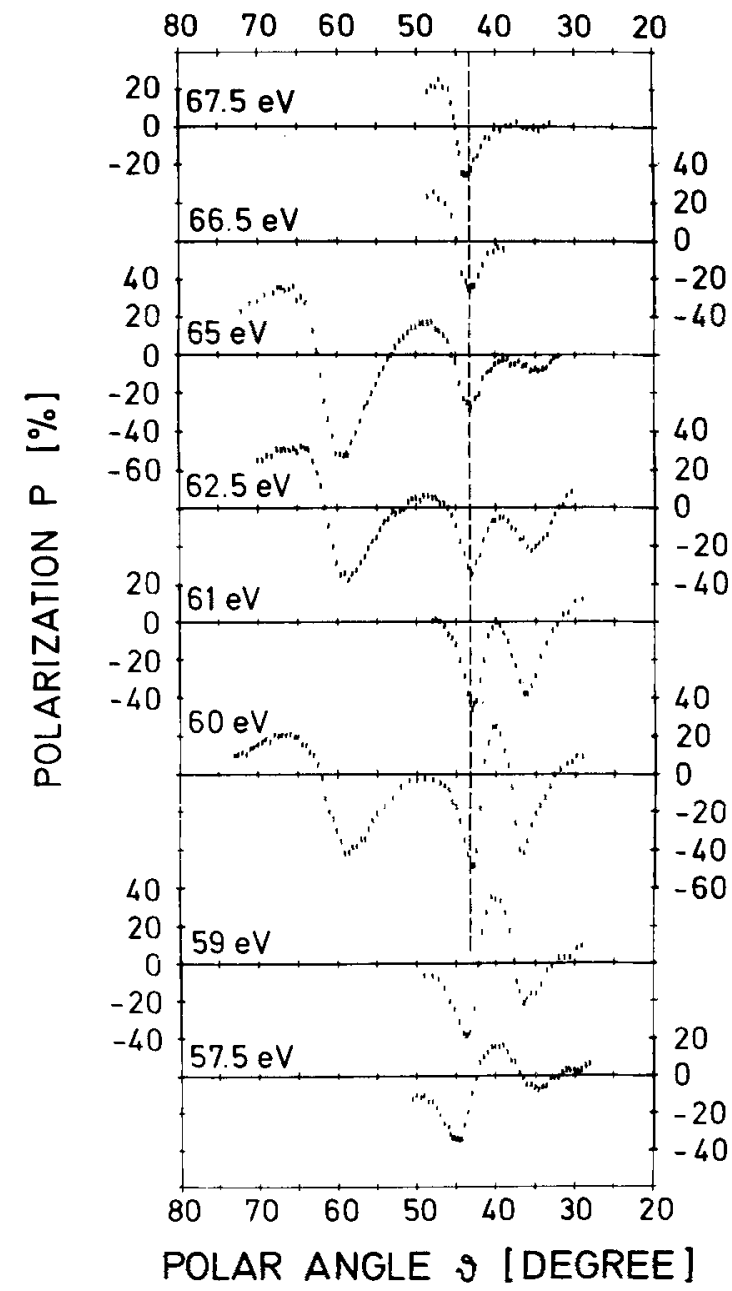

Fig. 9. The polarization $P$ as a function of the polar angle $\vartheta$ for $\varphi$ $=0^{\circ}$ in the energy range from $57.5 \mathrm{eV}$ to $67.5 \mathrm{eV}$

crystal lattice. After correction for the inner potential $\vartheta^{\prime}$ is equal to $38.5^{\circ}$. The direction of the electron beam characterized by $\vartheta^{\prime}=38.5^{\circ}$ and $\varphi=19^{\circ}$ thus nearly coincides with the [230] direction, which is characterized by $\tilde{\vartheta}=36^{\circ}$ and $\tilde{\varphi}=18.5^{\circ}$. It is interesting to note that the projection of the electron wavelength onto the surface normal is about half the layer spacing of the (111) planes in this energy range. Further investigations are required to get a deeper insight.

Although it is possible to interpret some polarization features, e.g. those described above, by analysis of the polarization data, it seems to be necessary, in general, to determine the spin-dependent intensities, as has been shown for W(001) by analyzing surface resonance effects [27]. From our measurements we only can evaluate the spin-up and spindown partial intensities $I_{\uparrow}, I_{\downarrow}$ of the diffracted beams, defined by 


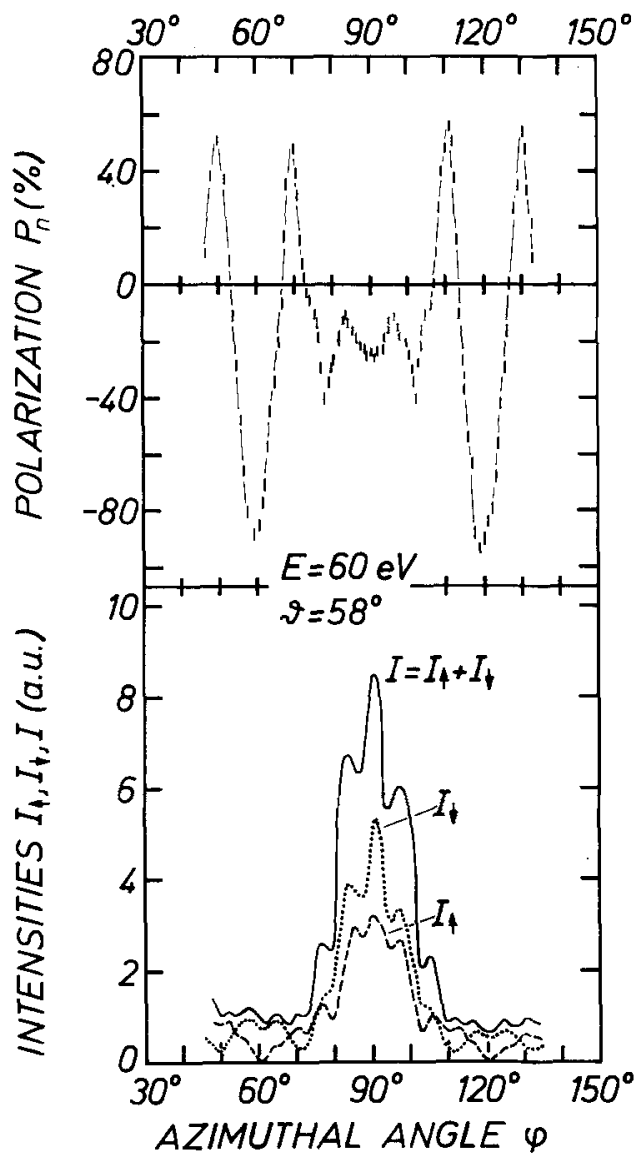

Fig. 10. Rotation diagrams of the polarization component $P_{n}$ the total intensity $I$ and the partial intensities $I_{\uparrow}$ and $I_{\downarrow}$ for $E=60 \mathrm{eV}$ and $\vartheta=58.5^{\circ}$

$I_{\uparrow}=I\left(1+P_{n}\right) / 2$

$I_{\downarrow}=I\left(1-P_{n}\right) / 2$

$I$ denotes the total intensity $I_{\uparrow}+I_{\downarrow}$ of the diffracted beam. Qualitatively, the partial intensities behave as the spin-dependent intensities of Ref. 27 [22]. In Fig. 10 we show an example, where at $E=60 \mathrm{eV}$ and $\vartheta$ $=58.5^{\circ}$ the polarization data $P_{n}(\varphi)$, and also the counting rate in the Mott detector (which is approximately proportional to the diffracted intensity [28]) are used to calculate the partial intensities $I_{\uparrow \downarrow}(\varphi)$ according to (5).

It can be seen from Fig. 10 that the strong maxima of the partial intensities around $\varphi=90^{\circ}$ lead to polarization of up to about $30 \%$, but the most interesting information of Fig. 10 is that the very strong variation of the polarization data around $\varphi=60^{\circ}$ and $\varphi=120^{\circ}$ have no counterpart in the total intensity, which only shows weak structure. This is understood by studying the partial intensities, which show quite different behaviour: the maxima of one partial intensity occur at the minima of the other, leading to a high degree of polarization combined with a remarkably high intensity.

Further, these minimum-maximum structures of the partial intensities compared to the total intensity clearly demonstrate a problem of LEED calculations: Due to the superposition of spin-up and spin-down intensity profiles, the total intensity has more peaks and less deep minima than each of the partial intensities. For any calculation treating the multiple scattering spin-independent (even as in [29]) the results will behave qualitatively similar to each of the partial intensities. Therefore such calculations will not give the correct superposed total intensity if strong spin effects are present, e.g. for high $Z$-materials.

\section{Conclusion}

The results presented in this paper demonstrate, that by investigation of spin-dependent effects in LEED additional information about the scattering crystal and the scattering processes are obtained. Especially partial intensity curves for up/down spin orientation show the serious influence of spin interactions on the total intensity of diffracted beams.

This work was sponsored by Deutsche Forschungsgemeinschaft, Sonderforschungsbereich 128. Financial support by Max-PlanckInstitut für Plasmaphysik is also gratefully acknowledged. Furthermore, the authors would like to thank Prof. Dr. D. Menzel for his continuous interest and many fruitful discussions, Dr. $\mathrm{K}$. Ertl for his valuable support and G. Nagleder for his technical help.

\section{References}

1. O'Neill, M.R., Kalisvaart, M., Dunning, F.B., Walters, G.K.: Phys. Rev. Lett. 34, 1167 (1975)

2. Kalisvaart, M., O'Neill, M.R.: Phys. Rev. B 17, 1570 (1978)

3. Müller, N., Wolf, D.: Bull. Am. Phys. Soc. II 21, 944 (1976)

4. Feder, R.: J. Phys. C 14, 2049 (1981)

5. Pierce, D.T., Celotta, R.G.: In: Advances in electronics and electron physics. Marton, C. (ed.), Vol. 56. New York: Academic Press 1981

6. Waller, G., Gradmann, U.: Phys. Rev. B 26, 6330 (1982)

7. Feder, R.: Surf. Sci. 63, 283 (1977)

8. Bauer, P., Feder, R., Müller, N.: Solid State Commun. 36, 249 (1980)

9. Kesmodel, L.L., Stair, P.C., Somorjai, G.C.: Surf. Sci. 64, 342 (1977)

10. Davies, J.A., Jackson, D.P., Norton, P.R., Posner, D.E., Unertl, W.N.: Solid State Commun. 34, 41 (1980)

11. Bøgh, E., et al.: In: Proc. $7^{\text {th }}$ Intern. Vacuum Congr. and $3^{\text {rd }}$ Intern. Conf. on Solid Surfaces, Vienna, 1977, p. A-2707

12. Veen, F. Van der, Smeenk, R.G., Tromp, R.M., Saris, F.W.: Surf. Sci. 79, 219 (1979)

13. Bauer, P., Feder, R., Müller, N.: Surf. Sci. 99, L 395 (1980) 
14. Adams, D.L., Nielsen, H.B., Hove, M.A. Van: Phys. Rev, B 20 , 4789 (1979)

15. Feder, R., Pleyer, H., Bauer, P., Müller, N.: Surf. Sci. 109, 419 (1980)

16. Klinken, J. van: Nucl. Phys. 75, 161 (1966)

17. Bauer, P., Eckstein, W., Müller, N.: (to be published)

18. Webb, M.B., Lagally, M.G.: Solid State Phys. 28, 301 (1978)

19. Kessler, J.: Polarized electrons. In: Texts an Monographs in Physics. Berlin, Heidelberg, New York: Springer Verlag 1976

20. Wang, G.C., Celotta, R.J., Pierce, D.T.: Surf. Sci. 119, 479 (1982)

21. Feder, R.: Surf. Sci. 68, 229 (1977)

22. Feder, R.: Phys. Lett. 78 A, 103 (1980)

23. Woodruff, D.P., Holland, B.W.: Phys. Lett. 31 A, 207 (1970)

24. Lagally, M.G., Ngoc, T.C., Webb, M.B.: Surf. Sci. 25, 444 (1971)

25. Farnsworth, H.E.: Phys. Lett. 36 A, 56 (1971)

26. Bauer, P.: Dissertation (PhD thesis) TU Munich, see also report IPP 9/34 (1980) of the Max-Planck-Institut für Plasmaphysik, Garching, Germany

27. McRae, E.G., Pierce, D.T., Wang, G.-C., Celotta, R.J.: Phys. Rev. B 24, 4230 (1981)
28. Feder, R., Müller, N., Wolf, D.: Z. Phys. B - Condensed Matter 28, 265 (1977)

29. Feder, R., Moritz, W.: Surf. Sci. 77, 505 (1978)

Peter Bauer

Institut für Experimentalphysik

Universität Linz

A-4040 Linz-Auhof

Austria

Wolfgang Eckstein

Max-Planck-Institut für Plasmaphysik

Euratom Association

D-8046 Garching bei München

Federal Republic of Germany

Norbert Müller

Fachbereich 4

Universität Osnabrück

D-4500 Osnabrück

Federal Republic of Germany 